\title{
Reverse Total Signed Vertex Domination in Graphs
}

\author{
Wensheng Li \\ Department of Mathematics and Information Sciences, Langfang Teachers College, Langfang, China \\ Email: wsli@live.cn
}

Received November 20, 2012; revised December 20, 2012; accepted December 27, 2012

\begin{abstract}
Let $G=(V, E)$ be a simple graph with vertex set $V$ and edge set $E$. A function $f: V \cup E \rightarrow\{-1,1\}$ is said to be a reverse total signed vertex dominating function if for every $v \in V$, the sum of function values over $v$ and the elements incident to $v$ is less than zero. In this paper, we present some upper bounds of reverse total signed vertex domination number of a graph and the exact values of reverse total signed vertex domination number of circles, paths and stars are given.
\end{abstract}

Keywords: Reverse Total Signed Vertex Domination; Upper Bounds; Complete Bipartite Graph

\section{Introduction}

In this paper we shall use the terminology of [1]. Let $G=(V, E)$ be a simple graph with vertex set $V(G)$ and edge set $E(G)$. Let $|V(G)|=n,|E(G)|=m$. For every $v \in V$, the open neighborhood of $v$, de- noted by $N_{G}(v)$, is a set $\{u \mid u v \in E\}$ and the closed neighborhood of $v$, denoted by $N_{G}[v]$, is a set $N_{G}(v) \cup\{v\}$. We write $d_{G}(v)$ for the degree of a vertex $v \in V(G)$ and the maximum and minimum degree of $G$ are denoted by $\Delta$ and $\delta$, respectively. For every $v \in V$, the edge-closed neighborhood of $v$, denoted by $N_{E}[v]$, is $N_{E}[v]=\{x \mid x=v$ or $x$ is incident to $v\}$.

Many domination parameters in graphs has been studied richly [2-4] A function $f: V \rightarrow\{-1,1\}$ is a signed dominating function if for every vertex

$$
v \in V, f(N[v])=\sum_{u \in N[v]} f(u) \geq 1 .
$$

The weight $\omega(f)$ of $f$ is the sum of the function values of all vertices in $G$. The signed domination number $\gamma_{s}(G)$ of $G$ is the minimum weight of signed dominating functions on $G$. This concept was introduced by Dunbar et al. [5] and has been studied by several authors [6-9]. As an extension of the signed domination, we give the definition of the reverse total signed vertex domination in a graph.

Definition 1. Let $G=(V, E)$ be a simple graph. A reverse total signed vertex dominating function of $G$ is a function $f: V \bigcup E \rightarrow\{-1,1\}$ such that $f\left(N_{E}[v]\right) \leq 0$ for all $v \in V$. The reverse total signed vertex domination number of $G$, denoted by $\gamma_{t s v}^{0}(G)$, is the maximum weight of a reverse total signed vertex dominating func- tion of $G$. A reverse total signed vertex dominating function $f$ is called a $\gamma_{t s v}^{0}$-function of $G$ if $w(f)=$ $\gamma_{t s v}^{0}(G)$.

\section{Properties of Reverse Total Signed Vertex Domination}

Proposition 1 For any graph $G$,

$$
\gamma_{\text {tsv }}^{0}(G)=(n+m)(\bmod 2) \text {. }
$$

Proof. Let $f$ be a $\gamma_{\text {tsv }}^{0}$-function of $G$. Then

$$
\gamma_{\text {tsv }}^{0}(G)=f(V)+f(E) \text {. }
$$

Let

$$
\begin{aligned}
& V_{1}=\{v \in V(G) \mid f(v)=1\}, \\
& V_{2}=\{v \in V(G) \mid f(v)=-1\}, \\
& E_{1}=\{e \in E(G) \mid f(e)=1\}, \\
& E_{2}=\{e \in E(G) \mid f(e)=-1\} .
\end{aligned}
$$

Then

$$
\begin{aligned}
\gamma_{t s v}^{0}(G) & =\left|V_{1}\right|-\left|V_{2}\right|+\left|E_{1}\right|-\left|E_{2}\right| \\
& =\left|V_{1}\right|-\left(n-\left|V_{1}\right|\right)+\left|E_{1}\right|-\left(m-\left|E_{1}\right|\right) \\
& =2\left|V_{1}\right|+2\left|E_{1}\right|-(n+m)
\end{aligned}
$$

Therefore $\gamma_{t s v}^{0}(G)=(n+m)(\bmod 2)$.

Propositon 2 For any graph $G, \gamma_{t s v}^{0}(G) \leq m$.

Proof. Let $f$ be a $\gamma_{t s v}^{0}$-function of $G$. Then for every $v \in V(G), \quad f\left(N_{E}[v]\right) \leq 0$ and we have 


$$
\begin{aligned}
0 & \geq \sum_{v \in V(G)} f\left(N_{E}[v]\right) \\
& =\sum_{v \in V(G)} f(v)+2 \sum_{u v \in E(G)} f(u v) \\
& =f(V)+2 f(E) .
\end{aligned}
$$

Thus $\gamma_{t s v}^{0}(G)=f(V)+f(E) \leq-f(E) \leq m$.

Propositon 3 For any graph $G, \gamma_{t s v}^{0}(G) \leq\lfloor n \Delta / 2\rfloor$.

Proof. Let $f$ be a $\gamma_{t s v}^{0}$-function of $G . V_{1}, V_{2}$, $E_{1}$ and $E_{2}$ are defined as Proposition 2. Then

$$
\gamma_{t s v}^{0}(G)=f(V)+f(E)=\left|V_{1}\right|-\left|V_{2}\right|+\left|E_{1}\right|-\left|E_{2}\right| .
$$

We define two induced graphs $G_{1}$ and $G_{2}$ of $G$ as follows:

$$
V\left(G_{1}\right)=V\left(G_{2}\right)=V(G), \quad E\left(G_{1}\right)=E_{1}, \quad E\left(G_{2}\right)=E_{2} .
$$

Then for every $v \in V\left(G_{1}\right)$,

$$
f\left(N_{E}[v]\right)=f(v)+d_{G_{1}}(v)-d_{G_{2}}(v) \leq 0
$$

and $d_{G_{1}}(v)-d_{G_{2}}(v) \leq-1$. For every $v \in V\left(G_{2}\right)$, we have

$$
f\left(N_{E}[v]\right)=f(v)+d_{G_{1}}(v)-d_{G_{2}}(v) \leq 0
$$

and $d_{G_{1}}(v)-d_{G_{2}}(v) \leq 1$. Thus

$$
\begin{aligned}
f(E) & =\left|E\left(G_{1}\right)\right|-\left|E\left(G_{2}\right)\right| \\
& =\frac{1}{2} \sum_{v \in V(G)} d_{G_{1}}(v)-\frac{1}{2} \sum_{v \in V(G)} d_{G_{2}}(v) \\
& =\frac{1}{2} \sum_{v \in V(G)}\left(d_{G_{1}}(v)-d_{G_{2}}(v)\right) \\
& =\frac{1}{2}\left(\sum_{v \in V_{1}}\left(d_{G_{1}}(v)-d_{G_{2}}(v)\right)\right. \\
& \left.+\sum_{v \in V_{2}}\left(d_{G_{1}}(v)-d_{G_{2}}(v)\right)\right) \\
& \leq \frac{1}{2}\left(\left|V_{2}\right|-\left|V_{1}\right|\right) .
\end{aligned}
$$

Therefore

$$
\begin{aligned}
\gamma_{\text {tsv }}^{0}(G) & =\left|V_{1}\right|-\left|V_{2}\right|+f(E) \\
& \leq\left|V_{1}\right|-\left|V_{2}\right|+\frac{1}{2}\left(\left|V_{2}\right|-\left|V_{1}\right|\right) \\
& =\frac{1}{2}\left(2\left|V_{1}\right|-n\right) .
\end{aligned}
$$

Since

$$
\begin{aligned}
0 & \geq \sum_{v \in V(G)} f\left(N_{E}[v]\right) \\
& =\sum_{v \in V(G)} f(v)+2 \sum_{u v \in E(G)} f(u v) \\
& =f(V)+2 f(E)=\left|V_{1}\right|-\left|V_{2}\right|+2 f(E) \\
& =\left|V_{1}\right|-\left|V_{2}\right|+\sum_{v \in V(G)}\left(d_{G_{1}}(v)-d_{G_{2}}(v)\right) \\
& \geq\left|V_{1}\right|-\left|V_{2}\right|+n(0-\Delta) \\
& =2\left|V_{1}\right|-n-n \Delta
\end{aligned}
$$

we have $\left|V_{1}\right| \leq \frac{n+n \Delta}{2}$. Therefore $\gamma_{\text {tsv }}^{0}(G) \leq\lfloor n \Delta / 2\rfloor$.

Propositon 4 For any star $K_{1, n}, \quad \gamma_{t s v}^{0}\left(K_{1, n}\right)=1$.

Proof. Let $f$ be a $\gamma_{t s v}^{0}$-function. Let

$$
\begin{aligned}
& V\left(K_{1, n}\right)=\left\{v_{0}, v_{1}, v_{2}, \cdots, v_{n}\right\}, \\
& E\left(K_{1, n}\right)=\left\{v_{0} v_{1}, v_{0} v_{2}, v_{0} v_{3}, \cdots, v_{0} v_{n}\right\},
\end{aligned}
$$

where $v_{0}$ is the center of $K_{1, n}$. Since for every $v \in V\left(K_{1, n}\right), \quad f\left(N_{E}[v]\right) \leq 0$, we have

$$
\begin{aligned}
\gamma_{\text {tsv }}^{0}\left(K_{1, n}\right) & =f(V)+f(E) \\
& =\sum_{i=1}^{n} f\left(N_{E}\left[v_{i}\right]\right)+f\left(v_{0}\right) . \\
& \leq 0+f\left(v_{0}\right) \leq 1
\end{aligned}
$$

On the other hand, consider the function

$$
g: \quad V\left(K_{1, n}\right) \cup E\left(K_{1, n}\right) \rightarrow\{-1,1\},
$$

such that

$$
g\left(v_{i}\right)=1(0 \leq i \leq n), \quad g\left(v_{0} v_{j}\right)=-1(1 \leq j \leq n) .
$$

Then $g$ is a reverse total signed vertex dominating function on $K_{1, n}$ and

$$
w(g)=g(V)+g(E)=1+n-n=1 .
$$

Thus $\gamma_{t s v}^{0}\left(K_{1, n}\right) \geq w(g)=1$, which implies that $\gamma_{\text {tsv }}^{0}\left(K_{1, n}\right)=1$.

Propositon 5 For any circle $C_{n}, \gamma_{t s v}^{0}\left(C_{n}\right)=0$.

Proof. Let $f$ be a $\gamma_{t s v}^{0}$-function of $C_{n}$. Let $V\left(C_{n}\right)=\left\{v_{1}, v_{2}, \cdots, v_{n}\right\}, E\left(C_{n}\right)=\left\{v_{1} v_{2}, v_{2} v_{3}, \cdots, v_{n} v_{1}\right\}$.

Since for every $v \in C_{n},\left|N_{E}[v]\right|=3$, we have

$$
f\left(N_{E}[v]\right) \leq-1 \text {. }
$$

Thus

$$
\begin{aligned}
& -n \geq \sum_{v \in V(G)} f\left(N_{E}[v]\right) \\
& =\sum_{v \in V(G)} f(v)+2 \sum_{u v \in E(G)} f(u v) . \\
& =f(V)+2 f(E)
\end{aligned}
$$

Therefore $\gamma_{t s v}^{0}\left(C_{n}\right)=f(V)+f(E) \leq-n-f(E) \leq 0$.

On the other hand, consider the mapping

$$
g: V\left(C_{n}\right) \cup E\left(C_{n}\right) \rightarrow\{-1,1\},
$$

such that

$$
g\left(v_{i}\right)=1(0 \leq i \leq n), g\left(e_{i}\right)=-1(1 \leq i \leq n) .
$$

Then $g$ is a reverse total signed vertex dominating function on $C_{n}$ and $w(g)=0$. Therefore

$$
\gamma_{t s v}^{0}\left(C_{n}\right) \geq w(g)=0,
$$

which implies $\gamma_{\text {tsv }}^{0}\left(C_{n}\right)=0$. 
Propositon 6 For any complete bipartite graph $K_{2, n}(n \geq 2), \gamma_{t s v}^{0}\left(K_{2, n}\right)=2-n$.

Proof. Let $f$ be a $\gamma_{t s v}^{0}$-function. Let

$$
\begin{aligned}
& U=\left\{u_{1}, u_{2}\right\}, V=\left\{v_{1}, v_{2}, \cdots, v_{n-1}, v_{n}\right\}, \\
& V\left(K_{2, n}\right)=U_{1} \cup V_{1}
\end{aligned}
$$

and

$$
E\left(K_{2, n}\right)=\left\{u_{1} v_{i}, u_{2} v_{i} \mid 1 \leq i \leq n\right\} .
$$

Since for every $v \in V,\left|N_{E}[v]\right|=3$, we have $f\left(N_{E}[v]\right) \leq-1$. Therefore

$$
\begin{aligned}
\gamma_{t s v}^{0}\left(K_{2, n}\right) & =f(V)+f(E) \\
& =\sum_{v \in V} f\left(N_{E}[v]\right)+f\left(u_{1}\right)+f\left(u_{2}\right) . \\
& \leq 2-n
\end{aligned}
$$

On the other hand, consider the mapping

$$
g: V\left(K_{2, n}\right) \cup E\left(K_{2, n}\right) \rightarrow\{-1,1\}
$$

such that $g\left(u_{1}\right)=g\left(u_{2}\right)=1, g\left(v_{j}\right)=1$ for $1 \leq j \leq n$, $g\left(u_{i} v_{j}\right)=-1$ for $i \in\{1,2\}$ and $1 \leq j \leq n$. Then $g$ is a reverse total signed vertex dominating function on $K_{2, n}$ and $w(g)=2-n$. Therefore $\gamma_{t s v}^{0}\left(K_{2, n}\right) \geq w(g)=2-n$, which implies $\gamma_{t s v}^{0}\left(K_{2, n}\right)=2-n$.

\section{Acknowledgements}

This work was supported by the Natural Science Foundation of Hebei Province (A2012408002), the Educational Commission of Hebei Province (ZH2011122, Z2011157) and Langfang Teachers College (LSZQ201106).

\section{REFERENCES}

[1] J. A. Bondy and V. S. R. Murty, "Graph Theory with Application,” Elsevier, Amsterdam, 1976.

[2] T. T. Chelvam and G. Kalaimurugan, "Bounds for Domination Parameters in Cayley Graphs on Dihedral Group,” Open Journal of Discrete Mathematics, Vol. 2, No. 1, 2012, pp. 5-10. doi:10.4236/ojdm.2012.21002

[3] T. W. Haynes, S. T. Hedetniemi and P. J. Slater, "Fundamentals of Domination in Graphs,” Marcel Dekker, New York, 1998.

[4] G. T. Wang and G. Z. Liu, "Rainbow Matchings in Properly Colored Bipartite Graphs,” Open Journal of Discrete Mathematics, Vol. 2, No. 2, 2012, pp. 62-64. doi:10.4236/ojdm.2012.22011

[5] J. E. Dunbar, S. T. Hedetniemi, M. A. Henning and P. J. Slater, "Signed Domination in Graphs," Combinatorics, Graph Theory, Applications, Vol. 1, 1995, pp. 311-322.

[6] O. Favaron, "Signed Domination in Regular Graphs," Discrete Mathematics, Vol. 158, No. 1, 1996, pp. 287293. doi:10.1016/0012-365X(96)00026-X

[7] Z. Zhang, B. Xu, Y. Li and L. Liu, "A Note on the Lower Bounds of Signed Domination Number of a Graph,” Discrete Mathematics, Vol. 195, No. 1, 1999, pp. 295-298. doi:10.1016/S0012-365X(98)00189-7

[8] X. Z. Lv, "Total Signed Domination Numbers of Graphs," Science in China A: Mathematics, Vol. 37, 2007, pp. 573578.

[9] X. Z. Lv, “A Lower Bound on the Total Signed Domination Numbers of Graphs," Science in China Series A: Mathematics, Vol. 50, 2007, pp. 1157-1162. 